\title{
Podal subspaces on the unit polydisk
}

\author{
by \\ KunYu Guo (Shanghai)
}

\begin{abstract}
Beurling's classical theorem gives a complete characterization of all invariant subspaces in the Hardy space $H^{2}(D)$. To generalize the theorem to higher dimensions, one is naturally led to determining the structure of each unitary equivalence (resp. similarity) class. This, in turn, requires finding podal (resp. s-podal) points in unitary (resp. similarity) orbits. In this note, we find that H-outer (resp. G-outer) functions play an important role in finding podal (resp. s-podal) points. By the methods developed in this note, we can assess when a unitary (resp. similarity) orbit contains a podal (resp. an s-podal) point, and hence provide examples of orbits without such points.
\end{abstract}

1. Introduction. Let $T$ be the unit circle in the complex plane, and let $L^{2}(T)$ be the Hilbert space of square integrable functions, with respect to arc-length measure. Recall that the Hardy space $H^{2}(D)$ over the open unit disk $D$ is the closed subspace of $L^{2}(T)$ spanned by the non-negative powers of the coordinate function $z$. If $M$ is a (closed) subspace of $H^{2}(D)$ that is invariant for the multiplication operator $M_{z}$, then Beurling's theorem says that there exists an inner function $\phi$ such that $M=\phi H^{2}(D)$. The reason for the success of Buerling's theorem is that the function theory in this case is one-dimensional and the associated polynomial ring in one variable is a principal ideal domain. Almost everyone who has thought about this topic must have considered the corresponding problem for $H^{2}$ on the polydisk. Let $M$ be a closed subspace of $H^{2}\left(D^{n}\right)$. We say that $M$ is an invariant subspace if $p M \subseteq M$ for any complex polynomial $p$ in $n$ variables. It is easy to see that $M$ is invariant if and only if it is invariant under multiplication by the coordinate functions $z_{1}, \ldots, z_{n}$. However in the higher dimensional case, one quickly sees that a Beurling-like characterization is not possible. From recent work [ACD, DPSY, DY, Guo1, Guo2, Yan1, Yan2], the other extreme appears to be true and hence one directs attention to investigating equivalence classes of invariant subspaces of $H^{2}\left(D^{n}\right)$ under some kind of equivalence relation.

2000 Mathematics Subject Classification: 46J15, 46H25, 47B35, 47B38.

This research was supported by NNSF (10171019) and the Shuguang project in Shanghai. 
Definition. Let $M_{1}, M_{2}$ be two invariant subspaces of $H^{2}\left(D^{n}\right)$. We say that they are unitarily equivalent (resp. similar) if there exists a unitary operator (resp. an invertible operator) $X: M_{1} \rightarrow M_{2}$ such that for any polynomial $p$,

$$
X p h=p X h, \quad \forall h \in M_{1} .
$$

To generalize Beurling's theorem to higher dimensions, one is naturally led to the following problems:

(1) give all unitary equivalence (resp. similarity) classes of invariant subspaces on the polydisk;

(2) determine the structure of each unitary equivalence (resp. similarity) class.

On the classical Hardy space $H^{2}(D)$, Beurling's theorem implies that there is only one unitary equivalence (resp. similarity) class. In the higher dimensional case, to determine the structure of unitary equivalence classes, Douglas and Yan [DY] introduced the following concept. An invariant subspace of $H^{2}\left(D^{n}\right)$ is called podal if every invariant subspace that is unitarily equivalent to it is contained in it. Furthermore, we say that an invariant subspace of $H^{2}\left(D^{n}\right)$ is s-podal if every invariant subspace that is similar to it is contained in it. Let $M$ be an invariant subspace. We use $\operatorname{orb}_{\mathrm{u}}(M), \operatorname{orb}_{\mathrm{s}}(M)$ to denote the unitary orbit and similarity orbit of $M$, respectively, that is, the unitary equivalence class and the similarity class of $M$. Obviously, $H^{2}\left(D^{n}\right)$ is a podal (resp. an s-podal) point in its unitary (resp. similarity) orbit. In [DY], Douglas and Yan introduced the condition ( $\star$ ) (to be recalled later in this paper), and proved that an invariant subspace which satisfies $(\star)$ is necessarily podal. In particular, they gave an example to show that not every unitary orbit contains a podal point. Note that every unitary (resp. similarity) orbit can contain at most one podal (resp. s-podal) point (if it exists) and hence the orbit is completely determined by its podal (resp. s-podal) point (if it exists). So a partial generalization of Beurling's theorem will be to find all podal (resp. s-podal) points.

In this note, we find that H-outer (resp. G-outer) functions play an important role in finding podal (resp. s-podal) points. This, in turn, is directly related to the factorization problem for $H^{2}\left(D^{n}\right)$ functions. By the methods developed in this note, we can assess when a unitary (resp. similarity) orbit contains a podal (resp. an s-podal) point, and hence provide examples of orbits without such points. In the next section, we will state and prove these results.

2. Podal subspaces on the unit polydisk. From Beurling's theorem, each nontrivial invariant subspace $M$ of the Hardy space $H^{2}(D)$ has a unique 
representation

$$
M=\eta B H^{2}(D)=\eta H^{2}(D) \cap B H^{2}(D),
$$

where $\eta$ is a singular inner function, and $B$ a Blaschke product. The "zeros" of the invariant subspace lying on the boundary $T$ depend on the singular part $\eta H^{2}(D)$. By [Gar], we know that the singular inner function $\eta$ is determined by the singular measure $d \sigma_{\eta}$ on $T$. Although higher dimensional Hardy invariant subspaces do not have the Beurling form, we can define the relevant analogue of singular distribution of invariant subspaces. Let $N\left(D^{n}\right)$ denote the Nevanlinna class defined in [Ru1]. For each $f \in N\left(D^{n}\right), f$ has radial limits $f^{*}$ a.e. on $T^{n}$. Moreover, there is a real singular measure $d \sigma_{f}$ on $T^{n}$ determined by $f$ such that the least harmonic majorant $u(f)$ of $\log |f|$ is given by

$$
u(f)=P_{z}\left(\log \left|f^{*}\right|+d \sigma_{f}\right),
$$

where $P_{z}$ denotes the Poisson integral on the unit polydisk. Use $N_{*}\left(D^{n}\right)$ to denote the class of all $f \in N\left(D^{n}\right)$ for which the functions $\log ^{+}\left|f_{r}\right|$ form a uniformly integrable family. Then for any $p>0, H^{p}\left(D^{n}\right) \subset N_{*}\left(D^{n}\right)$. By [Ru1, Th. 3.3.5],

$$
N_{*}\left(D^{n}\right)=\left\{f \in N\left(D^{n}\right): d \sigma_{f} \leq 0\right\} .
$$

For an invariant subspace $M$ of $H^{2}\left(D^{n}\right), Z_{\partial}(M)$ is the singular distribution of $M$ on $T^{n}$ defined by

$$
Z_{\partial}(M)=\inf \left\{-d \sigma_{f}: f \in M, f \neq 0\right\} .
$$

For two invariant subspaces $M_{1}, M_{2}$, the relation $Z_{\partial}\left(M_{1}\right) \leq Z_{\partial}\left(M_{2}\right)$ means that for each non-zero $f \in M_{2}$, there is a function $g \in M_{1}$ such that $-d \sigma_{g} \leq$ $-d \sigma_{f}$. As shown in $[\mathrm{DY}]$, the invariant $Z_{\partial}$ reveals many deep properties of invariant subspaces. Also in the same paper, Douglas and Yan introduced the condition $(\star)$ for invariant subspaces. An invariant subspace $M$ of $H^{2}\left(D^{n}\right)$ is said to satisfy the condition $(\star)$ if

(1) $h_{2 n-2}(Z(M))=0$ and

(2) $Z_{\partial}(M)=0$,

where $Z(M)=\left\{z \in D^{n}: f(z)=0, \forall f \in M\right\}$, and $h_{2 n-2}$ is the $(2 n-2)$ dimensional Hausdorff measure.

REMARK 2.1. From [Ru1, Th. 3.3.6], we see that for a function $f \in$ $H^{2}\left(D^{n}\right), d \sigma_{f}=0$ if and only if for almost all $w \in T^{n}$, the inner factor of the slice function $f_{w}(z)=f(w z)$ is a Blaschke product. Hence if there is such a function in $M$, then $Z_{\partial}(M)=0$. In particular, if $M \cap A\left(D^{n}\right) \neq\{0\}$, then $Z_{\partial}(M)=0$, where $A\left(D^{n}\right)$ is the polydisk algebra.

Now let us state Douglas and Yan's technical result [DY, Th. 1]. 
TheOREM 2.2 ([DY]). If $M$ satisfies the condition (1) in ( $\star$ ), and $\phi \in L^{\infty}\left(T^{n}\right)$, then $\phi M \subseteq H^{2}\left(D^{n}\right)$ if and only if $\phi \in N\left(D^{n}\right) \cap L^{\infty}\left(T^{n}\right)$ and $d \sigma_{\phi} \leq Z_{\partial}(M)$. Moreover, if $M$ satisfies $(\star)$, then $\phi M \subseteq H^{2}\left(D^{n}\right)$ if and only if $\phi \in H^{\infty}\left(D^{n}\right)$.

From [DY] or [Guo2], if $A: M_{1} \rightarrow M_{2}$ is a similarity (resp. unitary equivalence), then there is an invertible (resp. unimodular) function $\psi$ in $L^{\infty}(T)$ such that $A=M_{\psi}$. This says that similarity (or unitary equivalence) between invariant subspaces can be realized by an operator of multiplication by a bounded function. Combining this fact with Theorem 2.2, one sees that if an invariant subspace $M$ satisfies the condition $(\star)$, then $M$ is a podal (resp. s-podal) point in its unitary (resp. similarity) orbit. That is, $M$ is the maximum in its unitary (resp. similarity) orbit. Although each podal subspace $M$ is the maximum in its unitary orbit $\operatorname{orb}_{\mathrm{u}}(M)$, we give an example to show that not every unitary orbit has a podal point. For $f \in H^{2}\left(D^{n}\right)$, let $[f]$ denote the invariant subspace generated by $f$. We also say that $[f]$ is a principal invariant subspace generated by $f$.

EXAmple 2.3. Considering the Hardy space $H^{2}\left(D^{2}\right)$, it is easy to verify that the invariant subspaces $\left[z+\frac{1}{2} w\right]$ and $\left[w+\frac{1}{2} z\right]$ are unitarily equivalent because $\left|z+\frac{1}{2} w\right|=\left|w+\frac{1}{2} z\right|$ on $T^{2}$. Assume that there is a podal point $M$ in the unitary orbit $\operatorname{orb}_{\mathrm{u}}\left(\left[z+\frac{1}{2} w\right]\right)$. Then

$$
\left[z+\frac{1}{2} w\right] \subseteq M \quad \text { and } \quad\left[w+\frac{1}{2} z\right] \subseteq M .
$$

Therefore $z, w \in M$. So, $M \supseteq M_{0}$, where $M_{0}=\left\{f \in H^{2}\left(D^{2}\right): f(0,0)=0\right\}$. Hence $M=M_{0}$ or $H^{2}\left(D^{2}\right)$. Note that

$$
\operatorname{rank}\left(\left[z+\frac{1}{2} w\right]\right)=1 \text { but } \operatorname{rank}\left(M_{0}\right)=2 .
$$

So, $M \neq M_{0}$. Since $\left[z+\frac{1}{2} w\right]$ and $H^{2}\left(D^{2}\right)$ are generated by homogeneous polynomials $z+\frac{1}{2} w$ and 1 , respectively, Theorem 2 of [Yan1] implies that $\left[z+\frac{1}{2} w\right]$ and $H^{2}\left(D^{2}\right)$ are not unitarily equivalent, and hence $M \neq H^{2}\left(D^{2}\right)$. We have thus shown that the unitary orbit $\operatorname{orb}_{\mathrm{u}}\left(\left[z+\frac{1}{2} w\right]\right)$ has no maximum.

Note that $z+\frac{1}{2} w$ has no zero on $T^{2}$. By [Yan1], the invariant subspace $\left[z+\frac{1}{2} w\right]$ is similar to $H^{2}\left(D^{2}\right)$, and hence $H^{2}\left(D^{2}\right) \in \operatorname{orb}_{\mathrm{s}}\left(\left[z+\frac{1}{2} w\right]\right)$. Therefore, the inclusion

$$
\operatorname{orb}_{\mathrm{u}}\left(\left[z+\frac{1}{2} w\right]\right) \subset \operatorname{orb}_{\mathrm{s}}\left(\left[z+\frac{1}{2} w\right]\right)
$$

is strict.

The following is a modification of the example given in [DY] to show that not every similarity orbit has an s-podal point.

EXAMPLE 2.4. From [Ru2], there exist two functions $f, g$ in $H^{2}\left(D^{2}\right)$ such that 
(1) $|f|=|g|$ a.e. on $T^{2}$;

(2) $f / g$ is not the quotient of two $H^{\infty}$ functions.

Then obviously $[f]$ and $[g]$ are similar. But there exists no maximum in $\operatorname{orb}_{\mathrm{s}}([f])$. If there were one, say $M$, then there would exist functions $\phi_{1}, \phi_{2} \in$ $H^{\infty}\left(D^{2}\right)$ which are bounded below on $T^{2}$ such that

$$
\phi_{1} M=[f], \quad \phi_{2} M=[g] .
$$

This implies that $\phi_{2}[f]=\phi_{1}[g]$. By [Yan1], there would exist $r \in N_{*}\left(D^{2}\right)$ satisfying

$$
\phi_{2} f r=\phi_{1} g .
$$

Note that the equality $|f|=|g|$ insures that $r$ is a bounded analytic function and hence contradicts the fact that $f / g$ is not the quotient of two $H^{\infty}$ functions.

Recall that a function $f$ in the Hardy space $H^{2}(D)$ is outer if and only if $|g| \leq|f|$ a.e. on $T$ implies $f \mid g$, that is, there exists a function $h \in H^{2}(D)$ such that $g=f h$. Equivalently, $f$ is outer if and only if for any $\phi \in L^{\infty}(T)$, $\phi f \in H^{2}(D)$ implies $\phi \in H^{\infty}(D)$. Motivated by this observation, in [Guo1], we introduced the concept of H-outer functions. A function $f \in H^{2}\left(D^{n}\right)$ is called H-outer if $|g| \leq|f|$ a.e. on $T^{n}$ implies $f \mid g$. Obviously the definition is equivalent to the statement that for any $\phi \in L^{\infty}\left(T^{n}\right), \phi f \in H^{2}\left(D^{n}\right)$ implies $\phi \in H^{\infty}\left(D^{n}\right)$. This is different from the concept of outer function in the sense of Rudin [Ru1]. A function $f$ is outer in the sense of Rudin (briefly, $R$-outer $)$ if $\log |f(z)|=P_{z}[\log |f|]$ for some $z \in D^{n}$ and hence for all $z \in D^{n}$. For convenience, we say that a function $f \in H^{2}\left(D^{n}\right)$ is separately outer if for each $i, f\left(z_{1}, \ldots, z_{i-1}, z, z_{i+1}, \ldots, z_{n}\right)$ is outer in $H^{2}(D)$ as a function of $z$ for a.e. $\left(z_{1}, \ldots, z_{i-1}, z_{i+1}, \ldots, z_{n}\right) \in T^{n-1}$. Obviously, in the case $n=1$, these concepts are identical. In the case $n>1$, in [Guo1], we proved

$\{$ all R-outers $\} \subseteq\{$ all separately outers $\} \subseteq\{$ all H-outers $\}$.

In fact, the first inclusion is strict; we do not know if the second inclusion is strict.

Remark 2.5. By Proposition 2.1 of [Ge] or Proposition 2.9 of [Guo2], for a polynomial $p$, if $p$ has no zero in $D^{n}$, then $p$ is R-outer. For a polynomial $p$ in two variables, if $p$ depends strictly on two variables, then $p$ is separately outer if and only if $Z(p) \cap(D \times T \cup T \times D)=\emptyset$. In fact, if the above condition is satisfied, then obviously $p$ is separately outer. Assume that $p$ is separately outer. If there is some $\left(z_{0}, w_{0}\right) \in D \times T$ such that $p\left(z_{0}, w_{0}\right)=0$, by slightly perturbing $w_{0}$ on $T$ and using the continuous dependence of the roots on the coefficients, one deduces that for each $w$ near $w_{0}$, there exists $z \in D$ such that $p(z, w)=0$. Hence if $p$ is separately outer, then 
$Z(p) \cap(D \times T \cup T \times D)=\emptyset$. Of course, one can extend this conclusion to the case of several variables.

For a polynomial $p$, we define the monomial $\lambda_{p}(z)=z_{1}^{k_{1}} \ldots z_{n}^{k_{n}}$, where $k_{l}$ is the maximum degree of $z_{l}$ which occurs in $p$ for $l=1, \ldots, n$.

Proposition 2.6. Let $p$ be an irreducible polynomial, and $Z(p) \cap D^{n}$ $\neq \emptyset$. If $p$ is H-outer, then $p=c \lambda_{p} \bar{p}$ on $T^{n}$, where $c$ is a constant with $|c|=1$.

Proof. We can write $p=\lambda_{p} \bar{r}$ on $T^{n}$, where $r$ is a polynomial. Then $r$ is irreducible. Since $p$ is H-outer, the equality $(r / \bar{r}) p=\lambda_{p} r$ forces that

$$
\frac{r}{\bar{r}}=\frac{\lambda_{p} r}{\lambda_{p} \bar{r}}=\frac{\lambda_{p} r}{p}
$$

is a rational inner function. Since $p$ is irreducible and $Z(p) \cap D^{n} \neq \emptyset$, Theorem 5.2.5 of [Ru1] implies that there exists a polynomial $q$ such that

$$
\lambda_{p} r=q p .
$$

From the equality $p=\lambda_{p} \bar{r}$, one sees that $|q|=1$ on $T^{n}$, and hence $q$ is a monomial. Consequently, the equality $\lambda_{p} r=q p$ implies that there exists a constant $c$ with $|c|=1$ such that $p=c r$. This yields the desired conclusion.

If an invariant subspace $M$ contains an H-outer function, then by Proposition 3.2 of [Guo1], $M$ is necessarily the s-podal point in its similarity orbit. The following theorem may be seen to be a partial converse to this statement.

TheOrem 2.7. Assume that an invariant subspace $M$ satisfies the condition $(\star)$. Then the invariant subspace $[f M]$ is s-podal if and only if $f$ is H-outer.

Proof. First assume that $f$ is H-outer, and an invariant subspace $N$ is similar to $[f M]$. Then there exists a $\phi \in L^{\infty}\left(T^{n}\right)$ which is bounded below on $T^{n}$ such that $\phi[f M]=N$. Theorem 2.2 thus implies that $\phi f \in H^{2}\left(D^{n}\right)$. So, $\phi \in H^{\infty}\left(D^{n}\right)$. This insures that $N$ is a subspace of $[f M]$. We conclude that if $f$ is H-outer, then $[f M]$ is s-podal.

Now let $[f M]$ be s-podal. If $\phi \in L^{\infty}\left(T^{n}\right)$ is such that $\phi f \in H^{2}\left(D^{n}\right)$, our aim is to prove that $\phi$ is in $H^{\infty}\left(D^{n}\right)$. Of course, we may assume that $1 / 2 \leq|\phi| \leq 1$ on $T^{n}$. Otherwise, we replace $\phi$ by $\phi+c$, where $c$ is a large positive constant. One thus establishes a similarity:

$$
M_{\phi}:[f M] \rightarrow[\phi f M], \quad M_{\phi^{-1}}:[\phi f M] \rightarrow[f M] .
$$

Since $[f M]$ is s-podal, we see that

$$
\phi[f M] \subseteq[f M] .
$$


It follows that for any natural $k, \phi^{k} f \in H^{2}\left(D^{n}\right)$. By using an ideal of Schneider [ACD, see the proof of Proposition 3], one can prove $\phi \in H^{\infty}\left(D^{n}\right)$. Here, we give a different proof. For every natural number $k$, set $g_{k}=\phi^{k} f$ and extend $\phi^{k}$ to those $z$ in $D^{n}$ where $f(z) \neq 0$ by defining $\phi^{k}(z)=g_{k}(z) / f(z)$. By induction, it is easy to see that for any $z \notin Z(f)$,

$$
\phi^{k}(z)=\left(\phi^{1}(z)\right)^{k} .
$$

Since $\left(\phi^{k} f\right)(z)=\phi^{k}(z) f(z)=P_{z}\left[\phi^{k} f\right]$, one sees that

$$
\left|\left(\phi^{1}(z)\right)^{k} f(z)\right| \leq P_{z}\left[\left|\phi^{k} f\right|\right] \leq P_{z}[|f|]
$$

for each natural $k$. This implies that $\left|\phi^{1}(z)\right| \leq 1$ for each $z \notin Z(f)$. Now the fact that $\phi(z)=\phi^{1}(z)$ is analytic in $D^{n}$ follows from Hartogs' theorem. Thus $\phi$ is a bounded analytic function in $D^{n}$. This shows that $f$ is H-outer. The proof is complete.

COROLlary 2.8. A principal invariant subspace $[f]$ is s-podal if and only if $f$ is H-outer.

To obtain the next corollary we need to recall some results on the polynomial ring $\mathcal{C}=\mathbb{C}\left[z_{1}, \ldots, z_{n}\right]$. Let $P$ be a prime ideal in $\mathcal{C}$. The height of $P$ is defined to be the maximal length $l$ of any strictly increasing chain of prime ideals

$$
0=P_{0} \subset P_{1} \subset \ldots \subset P_{l}=P .
$$

Since the polynomial ring is Noetherian every prime ideal has finite height and the height of an arbitrary ideal is defined to be the minimum of the heights of its associated prime ideals. For an ideal $I$, one has $\operatorname{dim}_{\mathbb{C}} Z(I)=$ $n-l$, where $l$ is the height of $I$ and $\operatorname{dim}_{\mathbb{C}} Z(I)$ the complex dimension of the zero variety of $I$ (cf. [DPSY] or [ZS]). When an ideal $L$ is of height at least 2 , this condition guarantees that the zero variety of $L$ is an $h_{2 n-2}$-null set. This means that $[L]$, the invariant subspace generated by $L$, satisfies the condition $(\star)$. Let $I$ be an ideal of $\mathcal{C}$. Since $\mathcal{C}$ is Noetherian, $I$ is generated by a finite number of polynomials. This implies that $I$ has the greatest common divisor $p$. So, $I$ can be uniquely written as $I=p L$ for some ideal $L$, which is called the Beurling form of $I$.

Proposition 2.9. Let $I$ be an ideal and $I=p L$ be its Beurling form. Then height $I=1$ if and only if $p \neq$ constant. Equivalently, height $I \geq 2$ if and only if the greatest common divisor $\operatorname{GCD}(I)$ is 1 . In particular, we have height $L \geq 2$.

Proof. The necessity comes from Lemma 3.4 of [Guo2]. Conversely, if $p \neq$ constant, then all elements in $I$ are divisible by $p$, and hence they are also divisible by some irreducible factor $q$ of $p$. Then the ideal $I$ is contained in the prime ideal generated by $q$. Since the height of this prime ideal is 1 , the height of $I$ is 1 . 
Combining Theorem 2.7 with Proposition 2.9, we have

Corollary 2.10. Let $I$ be an ideal and $I=p L$ be its Beurling form. Then $[I]$ is s-podal if and only if $p$ is H-outer.

TheOREM 2.11. Assume that an invariant subspace $M$ satisfies the condition $(\star)$. Then there exists an s-podal point in the similarity orbit $\operatorname{orb}_{\mathrm{s}}([f M])$ if and only if $f$ can be factorized as $f=\phi$ where $\phi$ is in $H^{\infty}\left(D^{n}\right)$, and bounded below on $T^{n}$, and $h$ is H-outer.

Corollary 2.12. Let $I$ be an ideal and $I=p L$ be its Beurling form. Then there exists an s-podal point in the similarity orbit $\operatorname{orb}_{\mathrm{s}}([I])$ if and only if $p=\phi h$ where $\phi$ is in $H^{\infty}\left(D^{n}\right)$, and bounded below on $T^{n}$, and $h$ is H-outer.

Proof of Theorem 2.11. First assume that $f$ has a factorization as described in Theorem 2.11. By Theorem 2.7, $\left[\phi^{-1} f M\right]$ is s-podal. Notice that

$$
M_{\phi}:\left[\phi^{-1} f M\right] \rightarrow[f M]
$$

is a similarity between $\left[\phi^{-1} f M\right]$ and $[f M]$. This says that there exists an s-podal point in the similarity orbit $\operatorname{orb}_{\mathrm{s}}([f M])$.

Conversely, if there exists an s-podal point in $\operatorname{orb}_{\mathrm{s}}([f M])$, say $N$, then there exists a $\phi \in H^{\infty}\left(D^{n}\right)$ which is bounded below on $T^{n}$ such that $[f M]=\phi N$. Theorem 2.7 implies that $\phi^{-1} f$ is H-outer in $H^{2}\left(D^{n}\right)$. So, $f$ has the desired factorization.

It is easy to see that each s-podal subspace is podal. We do not know if there exists a podal subspace $M$ which is not s-podal. This would mean that $M$ is the maximum in the unitary orbit $\operatorname{orb}_{\mathrm{u}}(M)$, but not in the similarity orbit $\operatorname{orb}_{\mathrm{s}}(M)$. If we consider a principal invariant subspace $[f]$, then $[f]$ is podal if and only if for every unimodular function $\eta$ (i.e. $|\eta|=1$ a.e. on $T^{n}$ ), the relation $\eta f \in H^{2}\left(D^{n}\right)$ implies $\eta$ is an inner function. Therefore, for a principal invariant subspace $[f]$, the above problem can be stated as follows: if $f$ has the property that for any $g \in H^{2}\left(D^{n}\right)$ with $|g|=|f|$ a.e. on $T^{n}$, $g$ is necessarily factorized as the product of $f$ and an inner function, is $f$ H-outer?

To clarify this problem, let us recall that on the unit disk, the AdamyanArov-Krein theorem says that for $\phi \in L^{\infty}$, if the coset $\phi+H^{\infty}(D)$ contains two functions of unit norm, then it contains a function $\psi$ such that $|\psi|=1$ almost everywhere on $T$ [Gar, Th. 4.3]. Supposee one could generalize the Adamyan-Arov-Krein theorem to the polydisk; then the problem mentioned above would have an affirmative answer. Indeed, if $\phi f \in H^{2}\left(D^{n}\right)$ (we may suppose $\left.\|\phi\|_{\infty}<1\right)$, then $\phi+H^{\infty}\left(D^{n}\right)$ contains two functions of unit norm, and hence contains a function $\eta$ such that $|\eta|=1$ a.e. on $T^{n}$. This leads to the desired conclusion. 
However, on the polydisk, the Adamyan-Arov-Krein theorem is not valid. Rudin constructed a counterexample on the unit ball $B_{n}$ of $\mathbb{C}^{n}, n>1$ (see [Ru3, Example 13.10]). It is easy to see that a slight modification yields a counterexample on the polydisk. However, this does not mean that the answer to the above problem is negative.

Let $f \in H^{2}\left(D^{n}\right)$. We say that $f$ is $G$-outer if for any $g \in H^{2}\left(D^{n}\right)$ with $|g|=|f|$ a.e. on $T^{n}, g$ is necessarily factorized as the product of $f$ and an inner function. Equivalently, $f$ is G-outer if for every unimodular function $\phi$, the relation $\phi f \in H^{2}\left(D^{n}\right)$ implies $\phi$ is an inner function.

Similarly to Theorem 2.11, we have

TheOREm 2.13. Assume that a subspace $M$ satisfies the condition $(\star)$. Then there exists a podal point in the unitary orbit $\operatorname{orb}_{\mathrm{u}}[f M]$ if and only if $f$ can be factorized as $f=\phi h$ where $\phi$ is an inner function and $h$ is G-outer.

Corollary 2.14. Let $I$ be an ideal and $I=p L$ be its Beurling form. Then there exists a podal point in the unitary orbit orb $_{\mathrm{u}}[I]$ if and only if $p=\phi h$ where $\phi$ is a rational inner function and $h$ is a G-outer rational function.

Proof. We only need to prove that $\phi$ is a rational inner function. From the equality $p=\phi h$, one sees that for almost all $w \in T^{n}$, the slice function $\phi_{w}(z)=\phi(z w)$ is a finite Blaschke product. The reason is that the slice function $p_{w}(z)$ is a polynomial in one variable. By [Ru1, Th. 5.2.4], we see that $\phi$ is a rational inner function, completing the proof.

Corollary 2.15. Let $p$ be an irreducible polynomial which is not a monomial, such that $p \neq c \lambda_{p} \bar{p}$ on $T^{n}$ for any constant $c$. If both $p$ and $\lambda_{p} \bar{p}$ have zeros in $D^{n}$, then the unitary orbit $\operatorname{orb}_{\mathrm{u}}([p])$ contains no podal point.

Proof. Indeed, since

$$
\operatorname{orb}_{\mathrm{u}}([p])=\operatorname{orb}_{\mathrm{u}}\left(\left[\lambda_{p} \bar{p}\right]\right)
$$

if $\operatorname{orb}_{\mathrm{u}}([p])$ contains a podal point, then Corollary 2.14 implies that there exist rational inner functions $\eta_{1}, \eta_{2}$ and rational G-outer functions $h_{1}, h_{2}$ such that

$$
\lambda_{p} \bar{p}=\eta_{1} h_{1}, \quad p=\eta_{2} h_{2} .
$$

It is easy to see that the equality $\left|h_{1}\right|=\left|h_{2}\right|$ on $T^{n}$ implies that $h_{1}=h_{2}$ (possibly up to a constant factor). Therefore,

$$
\frac{\lambda_{p} \bar{p}}{p}=\frac{\eta_{1}}{\eta_{2}}
$$


Let $\eta_{i}=p_{i} / q_{i}$, where $p_{i}, q_{i}$ are relatively prime polynomials for $i=1,2$. Then

$$
\lambda_{p} \bar{p} p_{2} q_{1}=p p_{1} q_{2} .
$$

By [Ru1, Th. 5.2.4], the $q_{i}$ have no zeros in $D^{n}$, and there exist monomials $\tau_{i}$ such that $p_{i}=\tau_{i} \bar{q}_{i}$ for $i=1,2$. Obviously, $\lambda_{p} \bar{p}$ is irreducible and relatively prime to $p$. Because $q_{1}$ has no zero in $D^{n}$, we see that $p$ divides $p_{2}$. This means that there exists a polynomial $r_{2}$ such that $p_{2}=p r_{2}$. One thus obtains the equality

$$
\lambda_{p} \bar{p} r_{2} q_{1}=p_{1} q_{2} .
$$

Note that $\lambda_{p} \bar{p}$ has zeros in $D^{n}$, and hence $\lambda_{p} \bar{p}$ divides $p_{1}$. That is, there is a polynomial $r_{1}$ such that $p_{1}=\lambda_{p} \bar{p} r_{1}$. So, $r_{2} q_{1}=r_{1} q_{2}$. Since $q_{2}$ and $p_{2}$ are relatively prime, $q_{2}$ and $r_{2}$ are relatively prime. This implies that $q_{2}$ divides $q_{1}$. Similarly, $q_{1}$ divides $q_{2}$. We thus conclude that there is a constant $c$ such that $q_{1}=c q_{2}$. It follows that

$$
\frac{\eta_{1}}{\eta_{2}}=\frac{\bar{c} \tau_{1}}{c \tau_{2}} .
$$

Consequently,

$$
\bar{c} \tau_{1} p=c \tau_{2} \lambda_{p} \bar{p} .
$$

Since $p$ and $\lambda_{p} \bar{p}$ are irreducible, and not monomial, the above equality implies that there exists a constant $c$ such that $p=c \lambda_{p} \bar{p}$. This contradicts the assumptions, and hence $\operatorname{orb}_{\mathrm{u}}([p])$ contains no podal point.

REMARK 2.16. Let $I$ be an ideal, and $I=p L$ be its Beurling form. If $p$ satisfies the assumptions in Corollary 2.15, the same reasoning implies that the unitary orbit $\operatorname{orb}_{\mathrm{u}}([I])$ contains no podal point.

EXAMPLE 2.17. We consider the principal invariant subspace $[z+w+\alpha]$ of $H^{2}\left(D^{2}\right)$, where $\alpha$ is a constant. If $\alpha=0$, then $z+w$ is separately outer, and hence by Corollary 2.8, $[z+w]$ is podal. If $|\alpha| \geq 2$, then by [Ge] or [Guo2], $[z+w+\alpha]=H^{2}\left(D^{2}\right)$. For each $\alpha$ with $0<|\alpha|<2$, there exists no podal point in the unitary orbit $\operatorname{orb}_{\mathrm{u}}([z+w+\alpha])$. Indeed, $z+w+\alpha$ satisfies the assumption of Corollary 2.15 for such $\alpha$.

Finally, let us look at the relation between Hankel operators and podal subspaces. For $\phi \in L^{\infty}\left(T^{n}\right)$, the Hankel operator $H_{\phi}: H^{2}\left(D^{n}\right) \rightarrow L^{2}\left(T^{n}\right) \ominus$ $H^{2}\left(D^{n}\right)$ with symbol $\phi$ is defined by

$$
H_{\phi} f=(I-P) \phi f, \quad \forall f \in H^{2}\left(D^{n}\right),
$$

where $P$ is the projection from $L^{2}\left(T^{n}\right)$ onto $H^{2}\left(D^{n}\right)$. It is easy to see that the kernel ker $H_{\phi}$ is an invariant subspace. However, in contrast to the case $n=1$, not every invariant subspace is the kernel of some Hankel operator. In the case $n>1$, there is an invariant subspace that is not contained in the kernel of any Hankel operator. An example is $M=\overline{(z+w) H^{2}\left(D^{2}\right)}$. Indeed, 
if $M \subseteq \operatorname{ker} H_{\phi}$, then $\phi(z+w) H^{2}\left(D^{2}\right) \subseteq H^{2}\left(D^{2}\right)$. Since $z+w$ is separately outer, $\phi$ is in $H^{\infty}\left(D^{2}\right)$, and hence $H_{\phi}=0$. In fact, this example implies that the following general conclusion holds.

Proposition 2.18. Let $M$ be an invariant subspace. Then $M$ is s-podal if and only if $M$ is not contained in the kernel of any Hankel operator.

Corollary 2.19. Let $M=\operatorname{ker} H_{\phi}$. If $\operatorname{orb}_{\mathrm{s}}(M)$ contains an s-podal point $N$, then $N=H^{2}\left(D^{n}\right)$.

Proof. Let $N$ be an s-podal point in $\operatorname{orb}_{\mathrm{s}}(M)$. Then there is an invertible function $\psi$ in $L^{\infty}\left(T^{n}\right)$ such that $M=\psi N$. Since $\psi N \subseteq N$, by the proof of Theorem 2.7, $\psi \in H^{\infty}\left(D^{n}\right)$. Also note that $N \subseteq \operatorname{ker} H_{\phi \psi}$. By Proposition 2.18, we see that $\phi \psi \in H^{\infty}\left(D^{n}\right)$. This implies that $\psi \in \operatorname{ker} H_{\phi}=M$. Therefore, $1 \in N$, and hence $N=H^{2}\left(D^{n}\right)$.

EXAmple 2.20. Let $f, g$ be as in Example 2.4. Take $M=\operatorname{ker} H_{f / g}$. Then $\operatorname{orb}_{\mathrm{s}}(M)$ contains no s-podal point. Indeed, if $N$ were one, then $N=H^{2}\left(D^{2}\right)$ by Corollary 2.19. It follows that $M=\phi H^{2}\left(D^{2}\right)$ for some $\phi \in H^{\infty}\left(D^{2}\right)$. Therefore $(f / g) \phi=\phi^{\prime} \in H^{\infty}\left(D^{2}\right)$. This contradicts the fact that $f / g$ is not the quotient of two $H^{\infty}$ functions. For a similar reason, $\operatorname{orb}_{\mathrm{u}}(M)$ contains no podal point.

Acknowledgments. The author wishes to thank Prof. R. G. Douglas for his suggestions and encouragement. The author is also grateful to the referee for numerous suggestions which made this paper more readable.

\section{References}

[ACD] O. P. Agrawal, D. N. Clark and R. G. Douglas, Invariant subspaces in the polydisk, Pacific J. Math. 121 (1986), 1-11.

[AM] M. F. Atiyah and I. G. MacDonland, Introduction to Commutative Algebra, Addison-Wesley, Menlo Park, CA, 1969.

[DPSY] R. G. Douglas, V. I. Paulsen, C. H. Sah and K. R. Yan, Algebraic reduction and rigidity for Hilbert modules, Amer. J. Math. 117 (1995), 75-92.

[DY] R. G. Douglas and K. R. Yan, On the rigidity of Hardy submodules, Integral Equations Oper. Theory 13 (1990), 350-363.

[Gar] J. B. Garnett, Bounded Analytic Functions, Academic Press, New York, 1981.

[Ge] R. Gelca, Rings with topologies induced by spaces of functions, Houston J. Math. 21 (1995), 395-405.

[Guo1] K. Y. Guo, Characteristic spaces and rigidity for analytic Hilbert modules, J. Funct. Anal. 163 (1999), 133-151.

[Guo2] - Equivalence of Hardy submodules generated by polynomials, ibid. 178 (2000), 343-371.

[Ru1] W. Rudin, Function Theory in Polydiscs, Benjamin, New York, 1969.

[Ru2] -, Invariant subspaces of $H^{2}$ on a torus, J. Funct. Anal. 61 (1985), 378-384.

[Ru3] - New Constructions of Functions Holomorphic in the Unit Ball of $C^{n}$, CBMS Regional Conf. Ser. in Math. 63, Amer. Math. Soc., 1986. 
[Yan1] K. R. Yan, Equivalence of homogeneous principal Hardy submodules, Indiana Univ. Math. J. 41 (1992), 875-883.

[Yan2] - Rigidity theorem and Beurling theorem, in: Proc. Sympos. Pure Math. 51, Part 2, Amer. Math. Soc., 1990, 367-369.

[ZS] O. Zariski and P. Samuel, Commutative Algebra, Vol. I, II, Van Nostrand, 1958/1960.

Department of Mathematics

Fudan University

Shanghai, 200433, People's Republic of China

E-mail: kyguo@fudan.edu.cn

Received May 18, 2000

Revised version July 10, 2001 